\title{
A Rare Cause of Unilateral Tinnitus
}

\author{
Nik Mohd Syukra Nik Abd Ghani ${ }^{\mathrm{a}, \mathrm{b}}$, Nik Adilah Nik Othman ${ }^{\mathrm{a}}$, Amran Mohamad \\ ${ }^{a}$ Department of Otorhinolaryngology Hospital Universiti Sains Malaysia Kubang Kerian Kelantan \\ ${ }^{b}$ Department of Otorhinolaryngology Hospital Sultanah Nur Zahirah Terengganu
}

\section{INTRODUCTION}

Tinnitus is not a disease per se but it is a symptom. It can be either subjective or objective type and the underlying cause varies such as tumour, infection, vascular abnormality or Meniere's disease. Foreign body in the external ear is a common presentation especially in children. However, foreign body in the middle ear manifested with only symptom of tinnitus is a rare and unexpected presentation. Foreign body in the middle ear are commonly due to iatrogenic cause such as complication of myringotomy and ventilation tube insertion surgery. There are reported cases of complications of myringotomy and ventilation tube insertion, and they typically presented with the ear infection manifestation, myringosclerosis or permanent perforation of tympanic membrane. Currently, there are only few reported cases in literatures of foreign body in the middle ear following complication of medial migration of ventilation tube into a middle ear with intact tympanic membrane.

\section{Case report}

Forty-five years old Malay female with underlying Type 2 diabetes mellitus and hypertension presented with history of pulsatile left ear tinnitus of 1 year duration. She described that the tinnitus was mild and intermittent, and it was not affecting her daily activities and was not associated with hearing impairment and vertigo. She had a history of ventilation tube insertion on the left ear 10 years ago. On physical examination, the patient was well and there was no abnormality seen at the external ear and neck region. Cranial nerves were intact. There was no mastoid tenderness, no neck nodes palpable and no temporomandibular joint tenderness elicited. Otoscopic examination revealed normal external ear canal with greenish to bluish mass at posteroinferior part behind an intact tympanic membrane (Figure1).

Corresponding author:

Dr Nik Mohd Syukra Nik Abdul Ghani (MD)

Department of Otorhinolaryngology,

Head and Neck Surgery

School of Medical Sciences,

Universiti Sains Malaysia Health Campus,

16150 Kubang Kerian Kelantan, Malaysia

Mobile no: +60139488737

Email: niksyukra@gmail.com

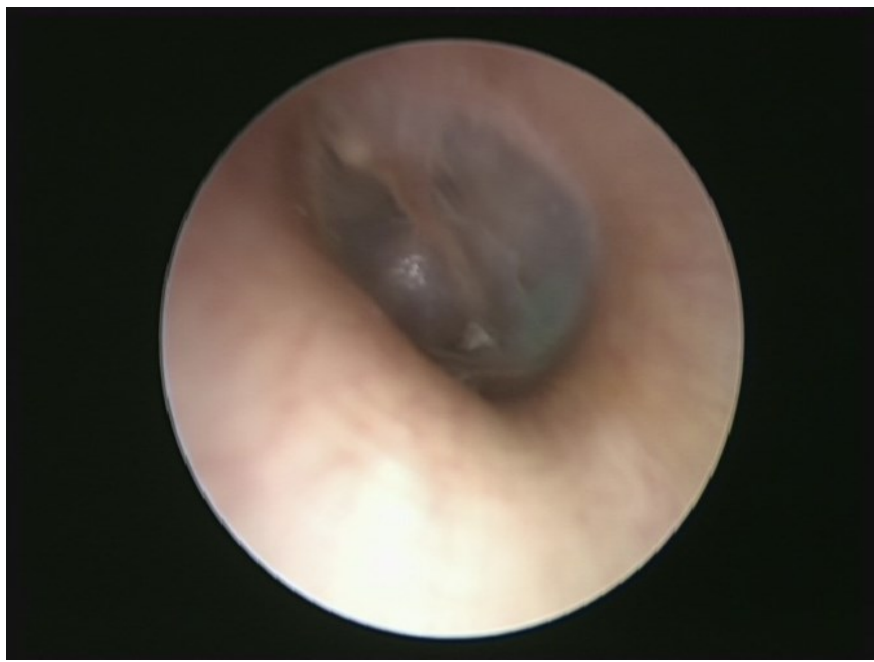

Figure 1: otoscopic view of left ear showing greenish-bluish mass at posteroinferior region behind the intact TM

However, neither the Brown's nor the Aquino's sign were appreciated. High resolution computed tomography scan of temporal bone showed the presence of a soft tissue mass occupying the left intratympanic region measuring about $7.7 \mathrm{~mm} \times 9.8 \mathrm{~mm} \times 4.6 \mathrm{~mm}$ (Figure2). The adjacent tympanic membrane and jugular plates were normal. The left ossicles were also visualized and normal. Both mastoid air cells were also aerated and clear. Following the imaging study, the diagnosis was concluded as suspicious foreign body ( $\mathrm{FB}$ ) in the middle ear with differential diagnosis of a glomus tympanicum. In view of the related past history of ventilation tube insertion, the diagnosis of foreign body in the middle ear was made. The patient underwent middle ear exploration surgery via permeatal approach with tympanomeatal flap under 
general anesthesia and the dislodged ventilation tube was found in the intratympanic cavity. Intraoperatively, the ventilation tube was removed successfully (Figure 3).

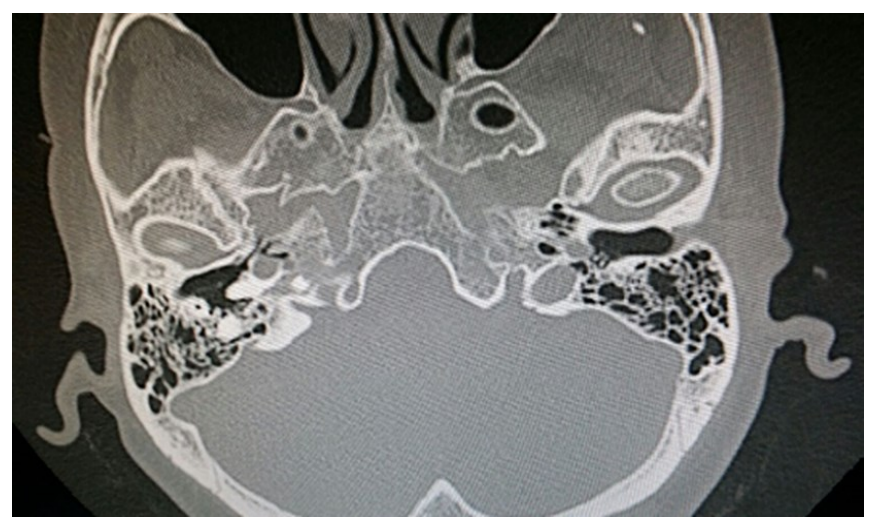

Figure 2: axial view of HRCT temporal bone showing well defined hypo density homogenous mass (arrow) at intratympanic region with no bony erosion

The patient was discharged well on day one postoperation without any complication. During follow up 2 weeks after the operation, the symptom of tinnitus had resolved completely.

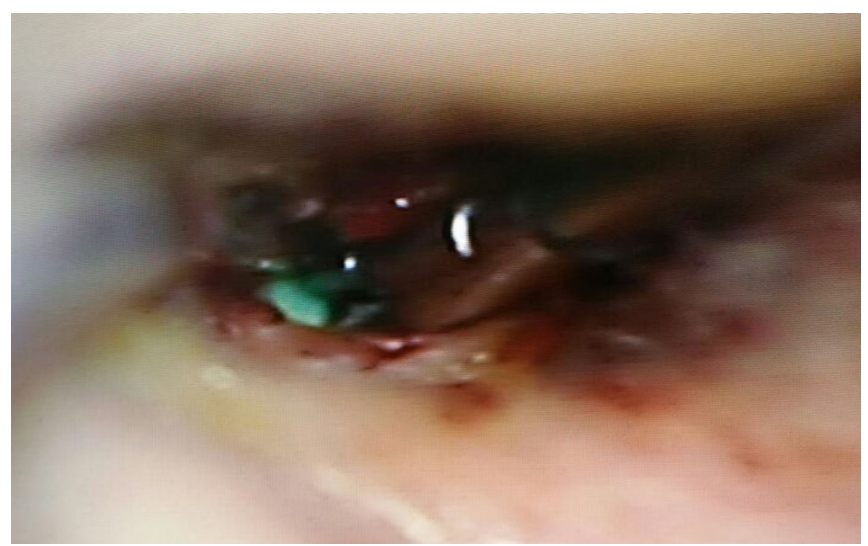

Figure 3: intraoperative findings showing greenish grommet tube in the middle ear and successfully removed

\section{DISCUSSION}

Tinnitus is not a disease per se but it is a symptom which is defined as a ringing, buzzing or hissing sensation in the ear in the absence of external sounds. It can either be subjective or objective tinnitus. ${ }^{1}$ Tinnitus has no gender predilection. Most individuals at one time or another may experience transient tinnitus lasting seconds or minutes whereas others may experience long-lasting tinnitus that can persist for a lifetime. The prevalence of tinnitus among those more than 65 years of age ranges from 12 to 15 percent $^{2}$ and the prevalence of tinnitus increases with age and peaks at age between 60 to 70 years old. One of the commonest cause of tinnitus is noise-induced hearing loss. Other causes include ear infections, high jugular bulbs, aberrant vessels in the middle ear, exposure to certain medications, middle ear tumour, ear infection and Meniere's disease. ${ }^{3}$ Tinnitus which is caused by foreign body in the middle ear is a rare presentation and not often reported. ${ }^{4}$

In otorhinolaryngology practices, myringotomy and ventilation tube insertion is one of the commonest procedures done throughout the year. It is a simple procedure and has minimal complication. According to a study done in Iran which look into complications of myringotomy and tympanostomy tube; myringosclerosis was found in $17.1 \%$, atrophy of the tympanic membrane in $1.2 \%$, permanent perforation of the tympanic membrane in $0.6 \%$ and tympanic membrane atelectasis in $0.6 \% .^{5}$ Although complication that may arise from myringotomy and ventilation tube insertion does not cause direct mortality, it may cause disturbance and may affect the quality of life of the patient.

Medial migration of ventilation tube is a rare complication of myringotomy and ventilation tube insertion ${ }^{6}$ and there were only a few reported cases. Medial migration of ventilation tube can be primary or secondary according to the time of it occurrence. There are few factors and risk factors that may contribute to the medial migration of ventilation tube such as technique of myringotomy, type of tube/grommet used and Eustachian tube dysfunction which cause negative pressure in the middle ear. ${ }^{6}$ Common presentation of suspicious complication of myringotomy and grommet insertion is patient with persistent or chronic otorrhea. Unlike in our case, patient manifested with a long history of myringotomy and grommet insertion and unilateral tinnitus without any sign of infection in the middle or external ear.

During clinical examination, otoscopy showed bluish -greenish mass behind an intact tympanic membrane which gives a few differential diagnosis such as glomus tumour, high jugular bulb, aberrant internal carotid artery and cholesterol granuloma. ${ }^{7}$ Hearing assessment is a tool that can help to narrow the diagnosis of a bluish mass behind intact tympanic membrane whether it is conductive or sensorineural hearing loss. The imaging study of choice which is high resolution computed tomography (HRCT) of temporal bone could help to establish the diagnosis and may rule out a glomus tumour. There are a few findings in HRCT of temporal bone that can be suggestive of glomus jugulotympanic tumor such as erosion of jugulotympanic plate or involvement of jugular foramen. Magnetic resonance imaging (MRI) study, however, has better advantage in differentiating glomus tumour as in T2-weighted images may show "salt and pepper" appearance of the glomus tumour. If the diagnosis is still unclear, a diagnostic angiography can be as confirmatory investigation which may show the feeding vessel of the paraganglioma. ${ }^{8}$ However, in this case the possibility of foreign body which was ventilation tube in the middle ear is more convincing in view of HRCT of 
temporal bone showing well-defined mass without any bony erosion and intact jugular foramen. Therefore no further MRI and angiogram were done.

Management of medial migration of ventilation tube can be conservative or interventive. There is a role of surgical intervention in a case of patient with recurrent or persistent otorrhea in which the tube has become a source of infection. The removal of ventilation tube in the middle ear can be achieved via permeatal, endaural or postauricular approach. The permeatal approach with tympanomeatal flap is associated with less morbidity, reduced operating time and faster healing. ${ }^{9}$ Our patient was discharge well after day 1 of surgery and during follow up, there was no immediate and long term complication.

Although foreign body in the middle ear is a rare cause of unilateral tinnitus, the sequalae of this condition might lead to more disastrous complication to the patient such as perilymphatic fistula. ${ }^{10}$ However, in this case, there was no major sequenced complication despite the presence of a long history of neglected foreign body in the middle ear.

\section{CONCLUSION}

In our case, we emphasized that physician should be aware of unusual presentation of unilateral tinnitus unaccompanied by infections may also be attributed by foreign bodies in the middle ear. Hence all aspect of unilateral tinnitus should be investigated thoroughly by $\mathrm{CT}$ scan to exclude all the relevant benign or malignant causes according to patient's history and clinical findings.

\section{REFERENCES}

1. Salvi R, Lobarinas E, Sun W. PHARMACOLOGICAL TREATMENTS FOR TINNITUS: NEW AND OLD. Drugs of the future. 2009;34(5):381-400.

2. Demeester K, van Wieringen A, Hendrickx JJ, Topsakal V, Fransen E, Van Laer L, et al. Prevalence of tinnitus and audiometric shape. B -ENT. 2007;3 Suppl 7:37-49.

3. Kreuzer PM, Vielsmeier V, Langguth B. Chronic Tinnitus: an Interdisciplinary Challenge. Deutsches Ã,,rzteblatt International.110(16):278 $-84$.

4. Eleftheriadou $A$, Chalastras $T$, Kyrmizakis $D$, Sfetsos S, Dagalakis K, Kandiloros D. Metallic foreign body in middle ear: an unusual cause of hearing loss. Head \& Face Medicine. 2007;3:23-.

5. Barati B, Hashemi SM, Goljanian Tabrizi A. Otological Findings Ten Years after Myringotomy with Tympanostomy Tube Insertion. Iranian Journal of Otorhinolaryngology. 2012;24(69):181 $-6$

6. Groblewski JC, Harley EH. Medial migration of tympanostomy tubes: an overlooked complication. International journal of pediatric otorhinolaryngology. 2006;70(10):1707-14.

7. Valvassori GE, Buckingham RA. Middle ear masses mimicking glomus tumors: radiographic and otoscopic recognition. Annals of Otology, Rhinology \& Laryngology. 1974;83(5):606-12.

8. Jehangir A, Pathak R, Shaikh B, Salman A, Fareedy SB, Qureshi A, et al. Jugulotympanic Paraganglioma: A Rare Cause of Vertigo. The American Journal of Case Reports. 2015;16:22831.

9. Kanona H, Virk JS, Owa A. Endoscopic ear surgery: A case series and first United Kingdom experience. World Journal of Clinical Cases : WJCC. 2015;3(3):310-7.

10. Hajiioannou J, Bathala S, Marnane C. Case of perilymphatic fistula caused by medially displaced tympanostomy tube. The Journal of Laryngology \& Otology. 2009;123(08):928-30. 
